\title{
Chimney reconstruction of the aortic arch in the Norwood procedure
}

\author{
Satoshi Asada, MD, ${ }^{\mathrm{a}}$ Masaaki Yamagishi, MD, PhD, ${ }^{\mathrm{a}}$ Keiichi Itatani, $\mathrm{MD}, \mathrm{PhD},{ }^{\mathrm{b}}$ and

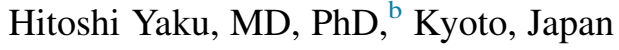

\footnotetext{
From the ${ }^{\mathrm{a} D e p a r t m e n t}$ of Pediatric Cardiovascular Surgery, Children's Medical Center, and ${ }^{\mathrm{b}}$ Division of Cardiovascular Surgery, Department of Surgery, Kyoto Prefectural University of Medicine, Kyoto, Japan.

Disclosures: Authors have nothing to disclose with regard to commercial support.

Received for publication Feb 12, 2017; revisions received April 13, 2017; accepted for publication April 27, 2017; available ahead of print June 24, 2017.

Address for reprints: Masaaki Yamagishi, MD, PhD, 465 Kajii-cho, Kamigyo-ku, Kyoto 602-8566, Japan (E-mail: myama@koto.kpu-m.ac.jp).

J Thorac Cardiovasc Surg 2017;154:e51-4

$0022-5223 / \$ 36.00$

Copyright $(2017$ by The American Association for Thoracic Surgery

http://dx.doi.org/10.1016/j.jtcvs.2017.04.079
}

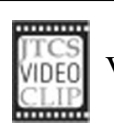

Video clip is available online.

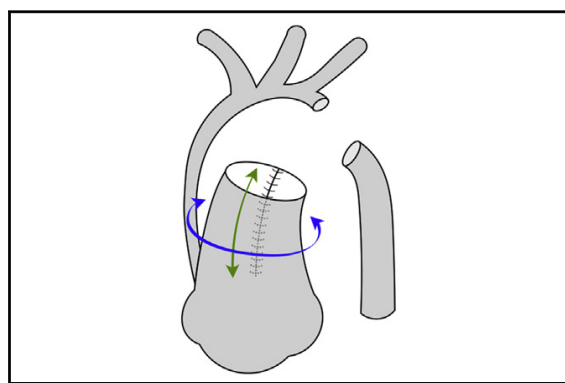

Chimney reconstruction of the arterial trunk in the Norwood procedure.

Central Message

We developed a novel "chimney reconstruction" technique for the Norwood procedure without patch supplementation, enabling low energy loss.

See Editorial Commentary page e55.
Narrowing of the aortopulmonary space and subsequent compression of the left pulmonary artery (PA) are critical problems in hypoplastic left heart syndrome (HLHS) occurring after the Norwood procedure. Although autologous aortic arch reconstruction is an ideal strategy, it can result in this complication and recoarctation. Future dilatation of the neo-aortic root followed by neo-aortic insufficiency is another critical complication. To avoid these complications without the use of patch supplementation, we developed a new aortic arch reconstruction technique for use in the Norwood procedure.

\section{CLINICAL SUMMARY}

A 2-month-old boy weighing $3.9 \mathrm{~kg}$ was diagnosed with HLHS with aortic atresia and mitral atresia complicated with patent foramen ovale, patent ductus arteriosus (PDA), and cor triatriatum. He underwent Norwood palliation, preceded by bilateral PA banding, cor triatriatum repair, and atrioseptectomy under cardiopulmonary bypass (CPB) at age 6 days.

After median resternotomy, an arterial cannula was placed in the 3.5-mm expanded polytetrafluoroethylene graft (W. L. Gore \& Associates, Flagstaff, Ariz) anastomosed to the right brachiocephalic artery. The other arterial cannula was placed directly in the descending aorta above the diaphragm to provide whole-body perfusion. Once CPB was established, the ductal tissue was completely resected. The right and left longitudinally arranged PA orifices (Figures 1, $A$ and $B$, and 2, step 1) were excised en bloc as a U-shaped cuff from the pulmonary trunk (Figure 2, step 2), instead of transecting the pulmonary trunk just beneath the bifurcation. The posterior U-shaped defect of the pulmonary trunk was closed longitudinally without any patch supplementation (Figure 2, step 3) and formed into a conical shape ("chimney reconstruction"). This maneuver enabled horizontal plication and longitudinal extension of the neo-aortic trunk (Figure 2, step 4). Subsequently, after removing the banding tapes from the PAs, the openings of the PAs were closed directly. The aortic arch and the descending aorta were partially anastomosed (Figure 2, step 5). The chimney-like neo-aortic trunk was anastomosed to this confluence (Figure 2, step 6). After cardiac arrest, the inner curvature of the ascending aorta was incised longitudinally. The corresponding neo-aortic trunk wall was also incised longitudinally. Both openings were anastomosed directly in a side-to-side fashion. A modified Blalock-Taussig shunt was constructed on the right side of the PA (Figure 1, $C$, and Video 1). The patient was subsequently weaned from CPB uneventfully, and his chest was closed after 5 days.

Postoperative CT demonstrated an adequately secured aortopulmonary space without aortic arch obstruction or compression of the left PA (Figure 1,D). No pressure gradient was found in the isthmus on the postoperative angiogram. Computational fluid dynamic (CFD) analysis of this neo-aortic arch showed laminar flow with small wall shear stress at the isthmus (Figure 1, E and $F$ ), 

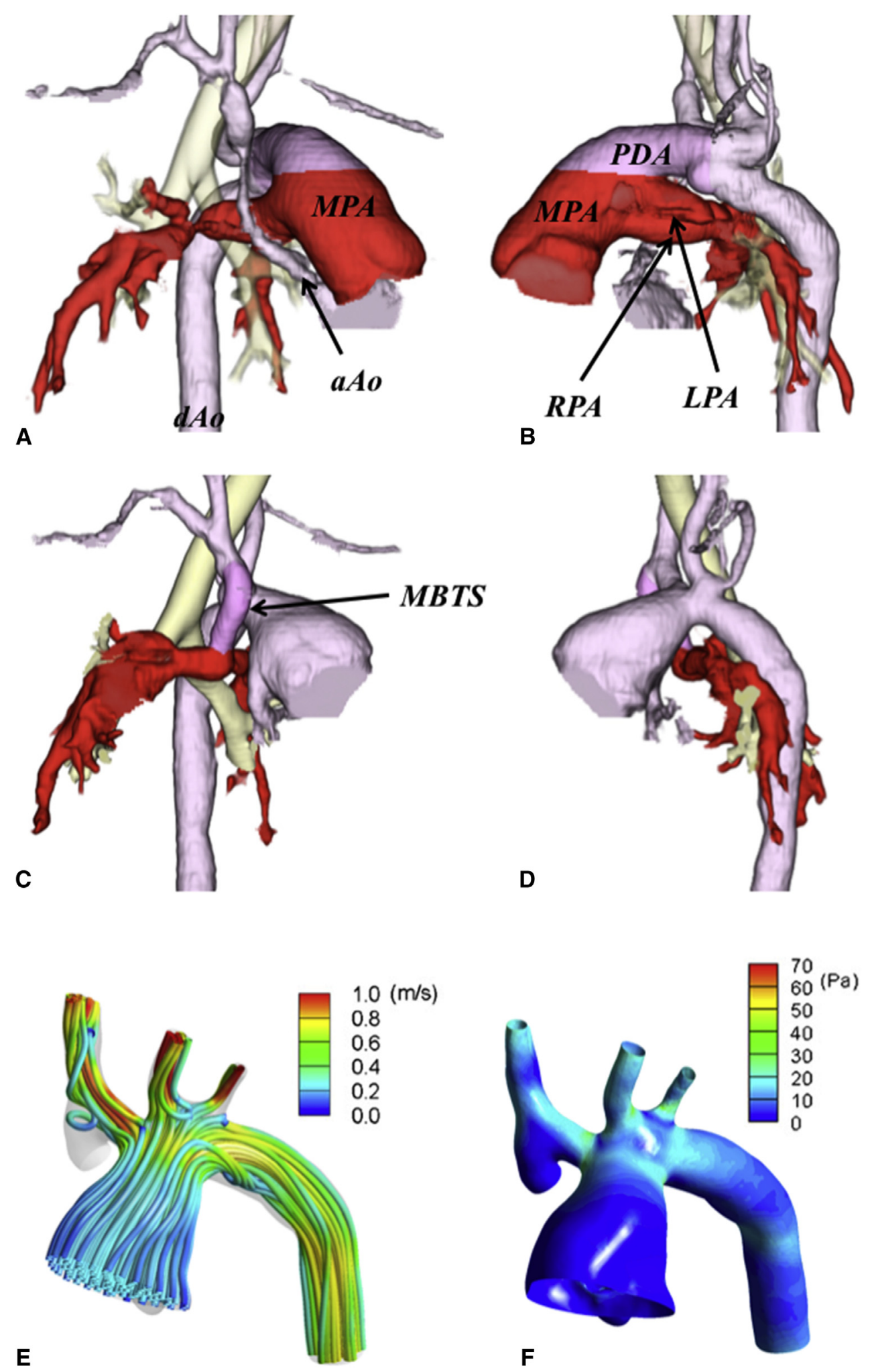

FIGURE 1. Preoperative and postoperative computed tomographic images and computational fluid dynamic analysis. A, (right oblique view) and (B) (left oblique view), Preoperative computed tomography (CT) scan showing a small ascending aorta and hypoplastic aortic arch, longitudinally-arranged branch pulmonary arteries, and banded bilateral pulmonary arteries. C, (right oblique) and (D) (left oblique), Postoperative CT showing the reconstructed neo-aortic arch securing a wide aortopulmonary space without compression of the left pulmonary artery. E, (streamlines) and (F) (wall shear stress), Computational fluid dynamic analysis after chimney reconstruction at the peak systolic phase. The isthmus of the neo-aorta develops little turbulent flow, and wall shear stress is minimal. The chimney reconstruction results in low energy loss. $a A o$, Ascending aorta; $d A o$, descending aorta; $M P A$, main pulmonary artery; $R P A$, right pulmonary artery; $L P A$, left pulmonary artery; $P D A$, patent ductus arteriosus; $M B T S$, modified Blalock-Taussig shunt. 
1
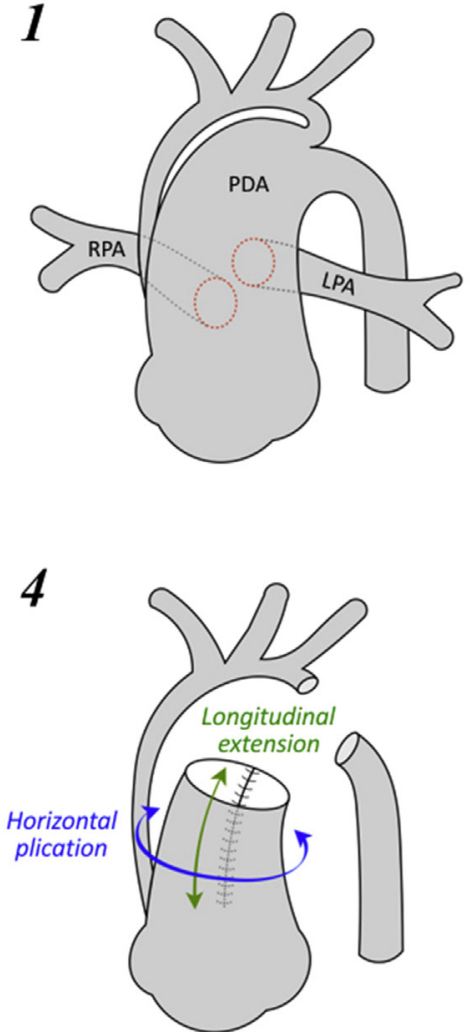

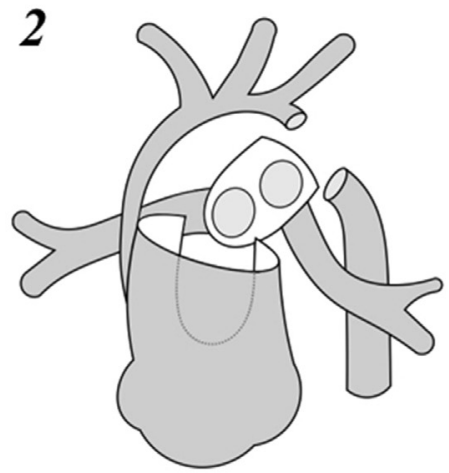

5

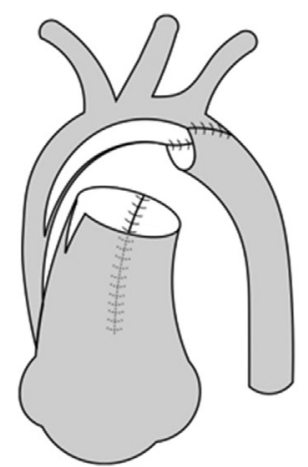

3

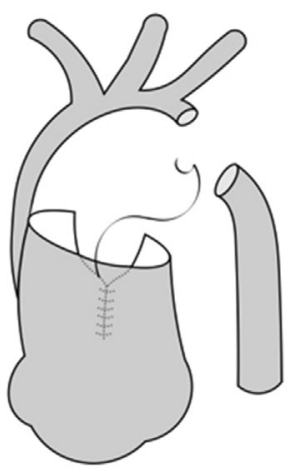

6

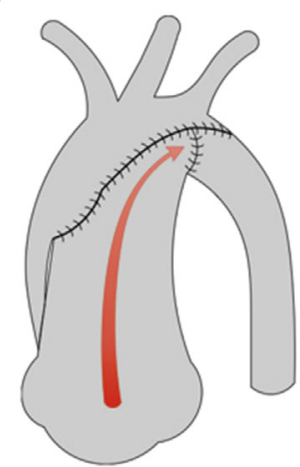

FIGURE 2. Chimney reconstruction. 1, Ductal tissue is completely resected. 2, Longitudinally-arranged pulmonary artery orifices are excised en bloc as a U-shaped cuff from the pulmonary trunk. 3, The posterior U-shaped defect of the pulmonary trunk is directly closed longitudinally. 4, This conical formation enables horizontal plication and longitudinal extension of the neo-aortic trunk. 5, The aortic arch is partially anastomosed to the descending aorta. 6, The neo-aortic trunk is directly anastomosed to the neo-aortic arch. The ascending aorta is anastomosed to the neo-aortic trunk in a side-to-side fashion. The bloodstream from the ventricle directed to the descending aorta is smooth. $R P A$, Right pulmonary artery; $P D A$, patent ductus arteriosus; $L P A$, left pulmonary artery.

resulting in low energy loss. The calculated energy loss of this neo-arch was very low (mean energy loss, $5.95 \mathrm{~mW}$ ). The patient underwent a bidirectional Glenn procedure at 8 months and a Fontan procedure at 2 years without any catheter interventions to either the PA or the neo-aortic arch.

\section{DISCUSSION}

Narrowing of the aortopulmonary space may cause postoperative compression of the left PA in the Norwood procedure. A late complication of neo-aortic root dilation that can cause this compression also has been observed. ${ }^{1}$ Both Baker's neo-aortic extension ${ }^{2}$ and Menon's posterior reduction of aortoplasty ${ }^{3}$ are effective salvage operations for releasing this compression. Our chimney reconstruction technique incorporates the benefits of these techniques, with longitudinal extension and horizontal plication of the pulmonary trunk accomplishing a wide aortopulmonary space, as well as preventing future dilatation of the neoaortic root. ${ }^{4}$

Another significant problem is recoarctation, which can result in future dysfunction of the right ventricle. Taking the potential for tissue growth into account, the use of supplementary materials should be avoided if possible. Patch materials themselves may lead to development of recoarctation due to degeneration and/or calcification. Our proposed chimney reconstruction technique can allocate a larger amount of tissue for aortic arch reconstruction, resulting in less tension and hence avoiding narrowing the aortopulmonary space. ${ }^{4}$ Moreover, the longitudinal, arrangement of the PA orifices frequently seen in this anomaly enabled us to create a tubular neo-aortic trunk without a steep caliber change. In cases where the PA bifurcation is arranged horizontally, an additional tiny $\mathrm{V}$-shaped incision is needed at the bottom edge of the posterior defect to prevent a drastic change in caliber after plication, ensuring that it does not extend to the sinotubular junction. This conical refashioning can contribute to avoiding turbulence and wall shear stress, as well as to the low energy loss of this neo-aortic arch on CFD analysis. ${ }^{5}$

This technique is widely applicable for various types of HLHS and its variants. Because most patients have a large and long pulmonary trunk, especially those who undergo bilateral PA banding, horizontal plication of the 


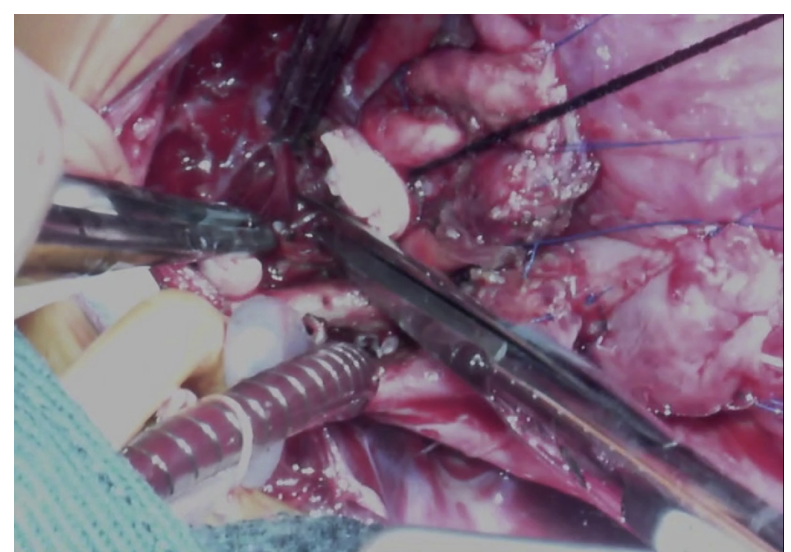

VIDEO 1. Chimney reconstruction of the aortic arch. After the cardiopulmonary bypass is started, the ductal tissue is removed completely. The longitudinally arranged pulmonary artery bifurcation is carefully detached, and this defect is trimmed into a U-shape. An additional tiny incision is made longitudinally to prevent an acute change in caliber after plication, ensuring that this does not extend to the sinotubular junction. The posterior defect is then sutured closed to complete the tubular trunk without using any patch material. This suture length is $12 \mathrm{~mm}$. Subsequently, the inner curvature of the arch is incised and partially anastomosed to the descending aorta. After induction of cardiac arrest, the tubular arterial trunk is anastomosed to this confluence. The inner curvature of the ascending aorta is opened near the root. The corresponding neo-aortic trunk wall is also incised longitudinally. The ascending aorta is anastomosed to the lateral wall of the arterial trunk. This completes the refashioning of the neoaortic arch. Finally, a modified Blalock-Taussig shunt is constructed at the right pulmonary artery. Video available at: http://www.jtcvsonline. org/article/S0022-5223(17)31101-7/addons.

pulmonary trunk can be performed without a concern for neo-aortic arch obstruction. This technique can be used as a primary Norwood procedure and is also appropriate for a short pulmonary trunk. In patients with a nondilated pulmonary trunk, care should be taken to avoid making the distal opening of the pulmonary trunk too small after closure of the posterior U-shaped defect. Left PA compression is an issue of concern, especially in patients with a long transverse aortic arch, because the pulmonary trunk needs to be pulled excessively to reach the descending aorta; however, in such patients the neo-aortic arch can be reconstructed without any supplementation using the combination of this chimney reconstruction of the pulmonary trunk with concomitant long side-to-side anastomosis between the transverse aortic arch and the descending aorta pulled more anterosuperiorly.

In summary, we have developed a novel alternative technique for aortic arch reconstruction in the Norwood procedure, especially in cases where homografts are not readily available. This technique allowed us to avoid compression of the left PA and to eliminate the issues of supplementary materials, enabling low energy loss.

\section{References}

1. Kanzaki T, Yamagishi M, Miyazaki T, Meada Y, Yaku H. Valve-sparing neoaortic root replacement late after the Norwood and Fontan procedures. Ann Thorac Surg. 2015;99:309-12.

2. Baker CJ, Wells WJ, Derby CA, Rizi S, Starnes VA. Ascending aortic extension for enlargement of the aortopulmonary space in children with pulmonary artery stenosis. Ann Thorac Surg. 2005;80:1647-51.

3. Menon A, Jones T, Barron D, Stumper O, Brawn W. Posterior reduction aortoplasty for left pulmonary artery compression after Norwood procedure. Ann Thorac Surg. 2011;91:1300-1.

4. Maeda Y, Yamagishi M, Miyazaki T, Yamamoto Y, Taniguchi S, Kanzaki T, et al. The treatment strategy for hypoplastic left heart syndrome. Kyobu Geka. 2014;67: 305-9 (Japanese).

5. Itatani K, Miyaji K, Qian Y, Liu JL, Miyakoshi T, Murakami A, et al. Influence of surgical arch reconstruction methods on single ventricle workload in the Norwood procedure. J Thorac Cardiovasc Surg. 2012;144:130-8. 